\title{
Early Recurrence of a Cardiac Malignant Fibrous Histiocytoma
}

\author{
Laura Reija López, Ángela Granda Bauza, Jose Manuel Martínez Cereijo, José Rubio Álvarez, \\ Belén Adrio Názar, Juan Sierra Quiroga \\ Complexo Hospitalario Universitario de Santiago de Compostela, A Coruña, Spain \\ Email: laurireija_lopez@hotmail.com
}

Received June 18, 2013; revised July 16, 2013; accepted July 30, 2013

Copyright (c) 2013 Laura Reija López et al. This is an open access article distributed under the Creative Commons Attribution License, which permits unrestricted use, distribution, and reproduction in any medium, provided the original work is properly cited.

\begin{abstract}
Malignant fibrous histiocytoma (MFH) is a rare tumor of the heart and the patients with these tumors usually have a poor prognosis. We report a case of MFH with an origin from the left superior pulmonary vein, involving the left atrium and protruding through the mitral valve, which needed urgent surgery. Complete resection was performed but local recurrence was detected one month later.
\end{abstract}

Keywords: Malignant Fibrous Histiocytoma; Early Recurrence

\section{Introduction}

Cardiac tumors are the cause of cardiac surgery approximately 1 in 500 cardiac surgical patients. Secondary or metastatic cardiac tumors are 30 times more common than the primary neoplasm. MFH is currently defined as a rare tumor. Despite several localizations having been described in the past, only exceptional cases primarily involve the heart. Local recurrence was found in $51 \%$ of patients who had a "complete" excision of the tumor. The treatment comprises surgery, chemotherapy and radiotherapy, although the survival time is usually considered to be less than 12 months [1].

\section{Description}

A 81-year-old woman, caucasian, with antecedents of hypertension, insuline dependent diabetes, paroxistic atrial fibillation, chronic pulmonary obstructive disease and frontal epidermic carcinome resected; presented to emergency with dyspnea and signs of heart failure since seven months ago. After transthoracic echocardiography, the patient was referred on admission our hospital for mass into left atrial and congestive heart failure. Then, she was arrived in room reanimation for acute pulmonary edema, hypoxia and atrial fibrillation. She needed orotracheal intubation and mechanical ventilation. Transesophageal echocardiography showed the presence of a cardiac mass involving the left atrium and protruding through the mi- tral valve (Figure 1). She was translated to operation room urgently.

The surgery was performed through a median sternotomy. Standard cardiopulmonary bypass was established, and the heart was arrested using cold antegrade blood cardioplegia. The right atrium was opened and through atrial septum, a solid mass attached firmly to left superior pulmonary vein and left atrium wall was revealed. This mass was excised completely. The mitral valve did not need repair. The excised tumor was taken to pathological examination. The patient was carried to reanimation room. She was hemodynamically unstable despite vasoactive drugs. 24 hours after the surgery, she was improved. The excised tumor was $6 \times 4.5 \times 4 \mathrm{~cm}$ in size, gray-white colored and $50 \mathrm{~g}$ of weigh, cystique, necrotic and hemorragic areas were seen in macroscopically exam (Figure 2).

Histopathological examination showed a pleomorphic proliferation of atypical spindle cells, admixed with scattered bizarre giant ones in a well-vascularized fribromyxoid stroma whith necrosis and hemorrhage. Inmunohistochemically staining the tumor cells were negative for: desmin, cytokeratin, HMB45, s100 protein, c-kit and myogenin. And they were positive for vimentine, smooth muscle actine and type IV collagen, with CD31 y CD34 markers.

Postoperative evolution was favorable. As a complication, she had a right pneumothorax requiring a thoracic 


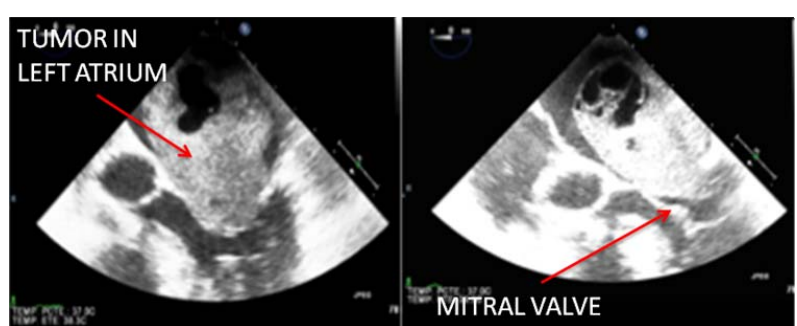

(a)

(b)

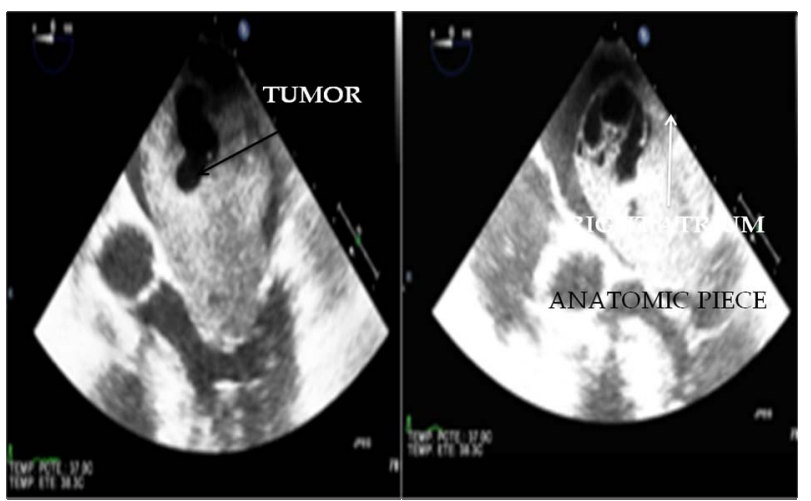

(c)

(d)

Figure 1. Transesophageal echocardiography showed a cardiac mass involving the left atrium and protruding through the mitral valve.

drain. She was discharged from hospital at twelve days after surgery. Despite total resection of all visible tissues and post-operative echocardiograph control without pathological findings; the recurrence of tumor at one month after surgery was showed in an ambulatory echocardiography and computerized tomography controls that showed the presence of a cardiac mass about $1.5 \mathrm{~cm}^{2}$, involving the left atrium and pulmonary veins (Figure 3 ). The patient didn't agree to surgical treatment again. At this time, five months after the surgery, the patient was free of symptoms.

\section{Discussion}

Cardiac tumors are divided into primary and secondary tumors. Primary cardiac tumors are very rare, with an autopsy incidence of $0.001 \%-0.03 \%$ [1]. Primary cardiac tumors include benign or malignant neoplasm that may arise from any tissue of the heart. Secondary or metastatic cardiac tumors are 30 times more common than the primary neoplasm with an autopsy incidence of $1.7 \%$ - 14\% [2]. Approximately $75 \%$ of primary cardiac tumors are benign, and 25\% are malignant. Approximately $50 \%$ of the benign tumors are myxomas, and about $75 \%$ of the malignant tumors are sarcomas [3]. The clinical incidence of these tumors is approximately 1 in 500 cardiac surgical patients.

Cardiac tumors cause symptoms in three separate

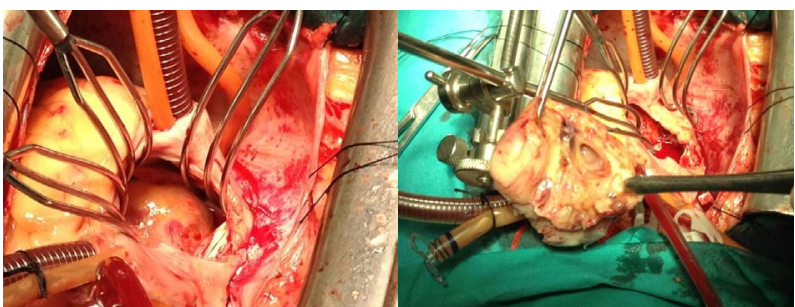

(a)

(b)

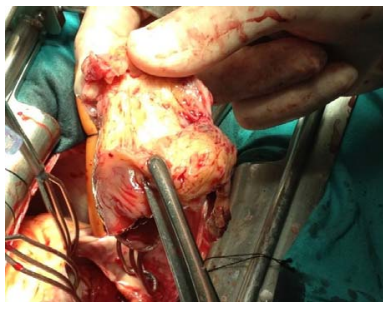

(c)

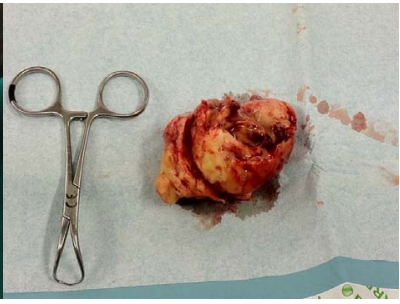

(d)
Figure 2. The right atrium wall was opened through atrial septum, a solid mass attached firmly to left superior pulmonary vein and left atrium wall was revealed and resected completely.

mechanisms: embolization, obstruction and arrhythmias. Embolization occurs frequently, fragments of the tumor itself can migrate, this may mimic endocarditis or vasculitis, and larger particles can cause cerebrovascular events. Atrial tumors, when large enough, may result in obstruction which impedes valvular flow, resulting in obstructive symptoms like syncope or congestive heart failure. Similarly, ventricular tumors may block outflow tracts resulting in chest pain, shortness of breath or syncopal episodes, as well as CHF. Finally, tumors can cause intramyocardial infiltration, and affect the conduction system resulting in heart block, arrhythmias and ventricular tachycardia, which may present as sudden death [4].

MFH is the most common soft tissue sarcoma in adults. A mixture of spindle cells in a storiform pattern, polygonal cells resembling histiocytes, and malignant giant cells characterizes it histologically. The cell of origin is the fibroblast or histioblast. MFH may be subclassified into fibrous, giant-cell, myxoid, and inflammatory variants. The fibrous variant accounted for two-thirds of the lesions. The prognosis is no different among the histologic subtypes. The depth of the tumor significantly affects survival, and three important groups were identified: superficial tumors, superficial tumors that recur in deep locations, and deeply situated tumors [5]. The use of immunohistochemistry is now essential in the diagnostic workup of any MFH-like tumor as it is now unacceptable to diagnose MFH based on morphology alone. In MFH an extensive immunohistochemistry panel is most likely required to exclude different lines of differentiation [6].

MFH usually occurs in the left atrium and often mimics myxoma. The tendency to metastasize early is not as 


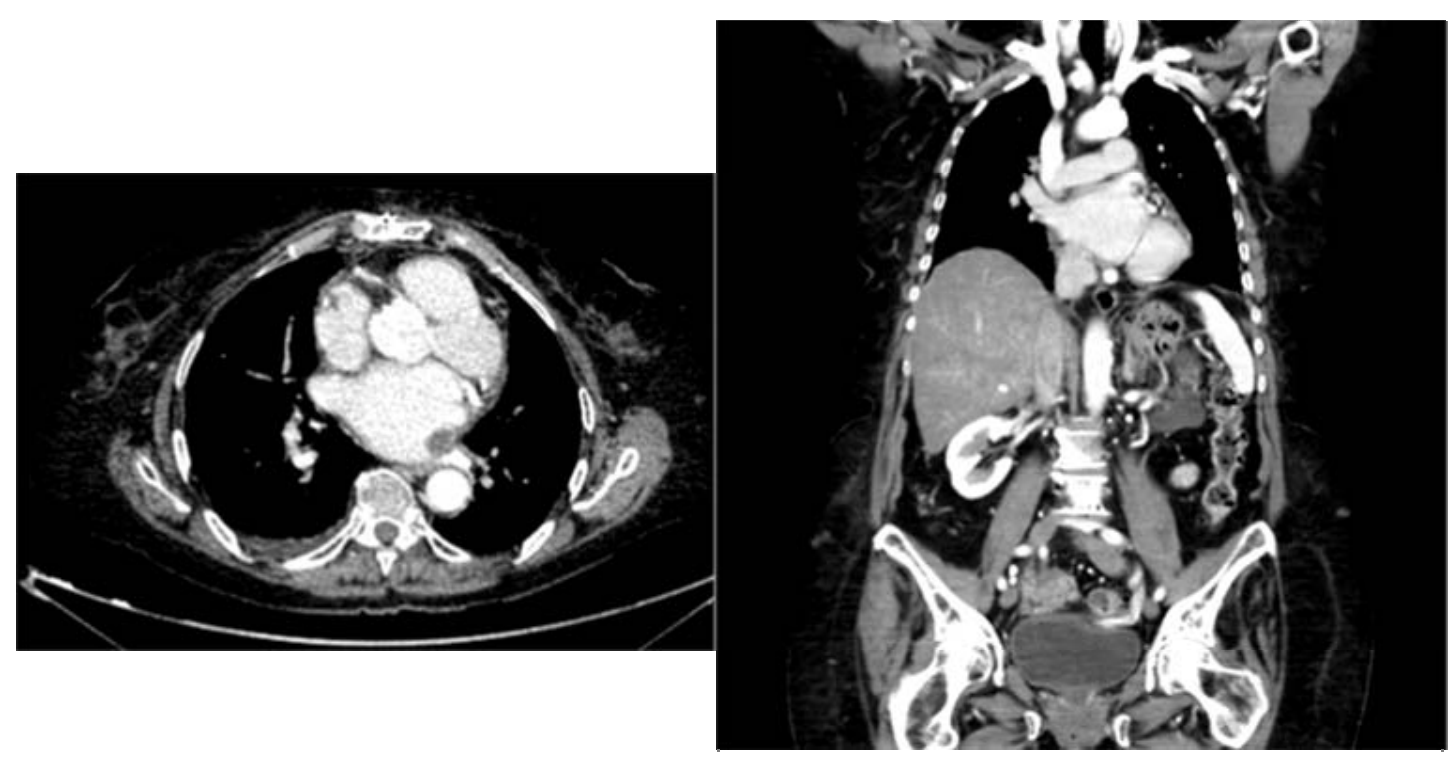

(a)

(b)

Figure 3. Filling defect identified by computerized tomography in left atrial arising left pulmonary vein, two months after surgery.

prominent as with angiosarcoma. Several reports exist documenting rapid symptomatic recurrence after incomplete resection despite chemotherapy. These patients often die of local cardiac disease prior to the development of metastases. If complete resection can be done at the original operation (particularly if the malignant nature is recognized) and adequate chemotherapy can be provided, survival may be improved. Incomplete resection is usually due to inadequate exposure of these broad-based tumors, which often extend to the anterior wall of the left atrium. Difficulty in exposing this posterior portion of the heart leads to inhibition of aggressive resection for clear margins and makes reconstruction difficult. These difficulties can be overcome by excising the heart and inverting it so that the posterior left atrium is now an anterior structure. This allows excellent visualization for aggressive resection and reconstruction done ex vivo, after which the heart is reimplanted [7].

MFH is currently defined as a rare tumor, and its diagnostic entity is continuously a subject of debate among pathologists. Despite several localizations having been described in the past, only exceptional cases primarily involve the heart. Local recurrence was found in $51 \%$ of patients who had a "complete" excision of the tumor [5] and the rate of metastasis was $23 \%$, but these was less likely when the tumor was small, superficially located, or had a prominent myxoid component [8].

Because of such high aggressiveness, the average survival time is usually considered to be less than 12 months. Despite the availability of valid chemotherapeutic regimens, the prognosis remains poor.

\section{Conclusion}

Malignant fibrous histiocytoma is a rare tumor and, in this case, the recurrence was found only one month after the complete resection.

\section{REFERENCES}

[1] R. Virmani, A. Burke and A. Farb, “Atlas of Cardio-VasCular Pathology,” Saunders, Philadelphia, 1996.

[2] A. Al-Mamgani, L. Baartman, M. Baaijens, et al., "Cardiac Metastases,” International Journal of Clinical Oncology, Vol. 13, No. 4, 2008, pp. 369-372. doi:10.1007/s10147-007-0749-8

[3] H. A. McAllister and J. J. Fenoglio Jr., "Tumors of the Cardiovascular System," Atlas of Tumor Pathology, Series 2, Armed Forces Institute of Pathology, Washington, 1978.

[4] L. Shapiro, "Cardiac Tumors: Diagnosis and Management,” Heart, Vol. 85, No. 2, 2001, pp. 218-222. doi:10.1136/heart.85.2.218

[5] M. M. Kearney, E. H. Soule and J. C. Ivins, "Malignant Fibrous Histiocytoma a Retrospective Study of 167 Cases,” Cancer, Vol. 45, No. 1, 2006, pp. 167-178. doi:10.1002/1097-0142(19800101)45:1<167::AID-CNCR 2820450127>3.0.CO;2-8

[6] O. M. Al-Agha and A. A. Igbokwe, "Malignant Fibrous Histiocytoma: Between the Past and the Present," Archives of Pathology \& Laboratory Medicine, Vol. 132, No. 6, 2008, pp. 1030-1035.

[7] L. H. Cohn, "Cardiac Surgery in the Adult," Third Edition, McGraw-Hill, Toronto, 2008.

[8] S. W. Weiss and F. M. Enzinger, "Myxoid Variant of 
Malignant Fibrous Histiocytoma,” Cancer, Vol. 39, No. 4, 1977, pp. 1672-1685.
doi:10.1002/1097-0142(197704)39:4<1672::AID-CNCR2 820390442>3.0.CO;2-C 